\title{
Editorial
}

Notfall Rettungsmed 2016 · 19:1-2

DOI 10.1007/s10049-016-0127-z

Online publiziert: 29. Januar 2016

๑) Springer-Verlag Berlin Heidelberg 2016

CrossMark
C.K. Lackner ${ }^{1} \cdot$ M. Reng ${ }^{2}$

${ }^{1}$ Drees \& Sommer AG, München, Deutschland

${ }^{2}$ Goldberg-Klinik, Med. Klinik II, Kelheim, Deutschland

\section{„Für tiefe Spuren braucht es Profil}

\section{Nachruf auf Prof. Dr. med. Heinzpeter Moecke}

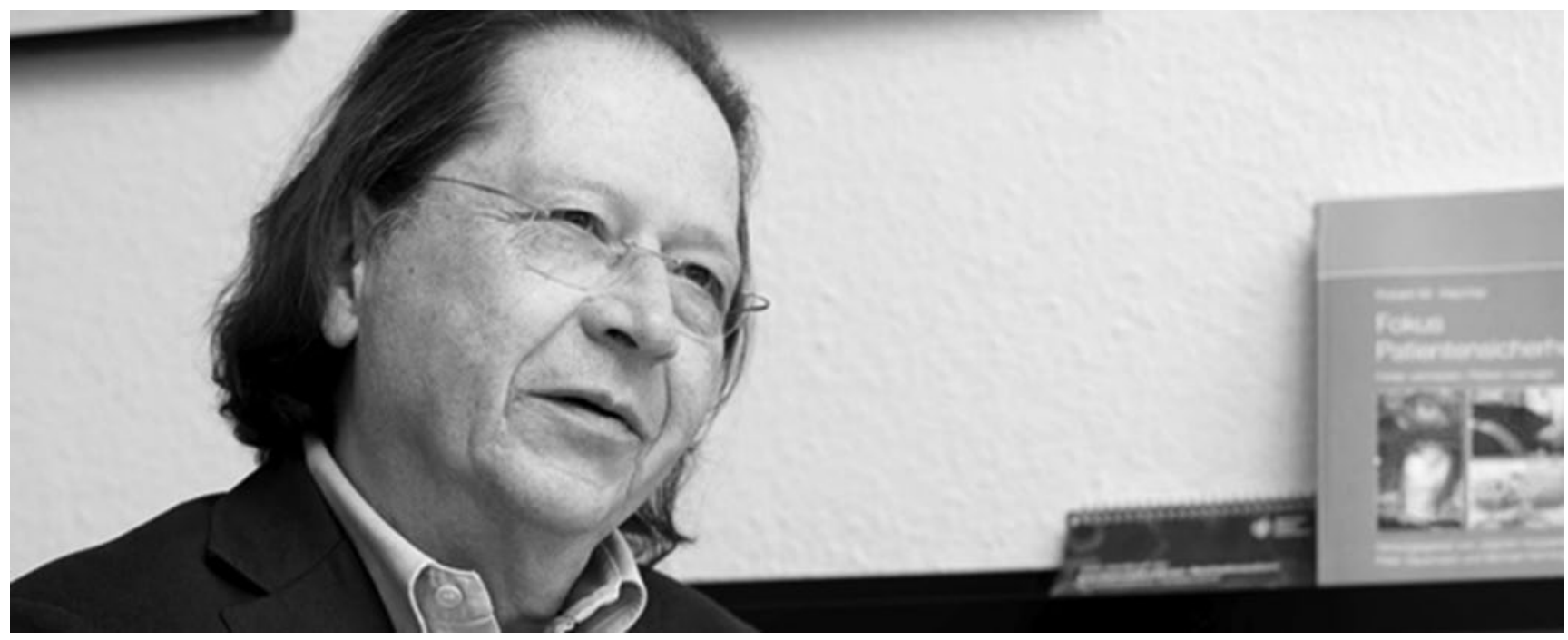

Prof. Dr. med. Heinzpeter Moecke ist am 26.11.2015 nach längerer Krankheit in Hamburg verstorben. Mit ihm verliert die Notfallmedizin in Deutschland einen kreativen und zugleich visionären Vordenker wie philanthropen Netzwerker, der auf seinem beruflichen Lebensweg unprätentiös wie leidenschaftlich - die Entwicklung der Notfallmedizin und des modernen Rettungswesens wesentlich vorangetrieben und nachhaltig geprägt hat.

Als einer der Gründungsherausgeber der Fachzeitschrift Notfall + Rettungsmedizin setzte er ab 1997 sehr früh wichtige und zukunftsweisende Akzente, u. a. mit Leitthemen zur Simulation in der Notfallmedizin und zur zentralen Notaufnahme.

Motiviert durch sein bis zuletzt breites Interesse sowie seine tief verwurzelte $\mathrm{Neu}$ - gier war er gleichermaßen Trendscout und Motor des Fortschritts. Sein unerschütterlicher Kompass für Interdisziplinarität und berufsübergreifendes Denken und Handeln machten ihn für viele zu einem sehr geschätzten Rat- und Ideengeber, der offen und verlässlich war, aber auch hartnäckig für seine Überzeugungen eintrat. Überzeugend durch Authentizität und inhaltlichen Tiefgang war sein Wort bei Fachveranstaltungen und in vielen Gremien gleichermaßen willkommen.

Prof. Moecke war langjährig in zahlreichen Verbandsfunktionen und Ehrenämtern aktiv, u. a. als Vorsitzender der Arbeitsgemeinschaft in Norddeutschland tätiger Notärzte (AGNN) und als Landesfeuerwehrarzt und Ärztlicher Leiter Rettungsdienst in Hamburg. Zudem war er Gründungsmitglied der deutschlandweit ersten Leitenden Notarztgruppe, die 1985 in Hamburg etabliert wurde. Er engagierte sich im Ausschuss für Notfallund Katastrophenmedizin der Bundesärztekammer und war über viele Jahre Mitglied der Sektion Rettungswesen der Deutschen Interdisziplinären Vereinigung für Intensiv- und Notfallmedizin (DIVI).

Prof. Moecke bereitete in Deutschland den steinigen Weg für eine einheitliche Dokumentation (bundeseinheitliches Notarztprotokoll), die er bis zuletzt weiterentwickelte. Seine Ideen zur Organisation der notfallmedizinischen Versorgung bei Großschadenslagen sind heute in Deutschland vielerorts gelebter Teil der etablierten Versorgungsstrategien. 2003 war er Mitgründer der Bundesarbeitsgemeinschaft Zentrale Notaufnahme (BAG-ZNA).

Heipe, wie ihn Freunde und Kollegen nennen durften, wurde am 15.08.1952 
in Berlin geboren. Von 1974 bis 1980 studierte er Medizin in Hamburg, um nach der Approbation und Promotion das amerikanische Staatsexamen und die Anerkennung als Facharzt für Anästhesiologie zu erwerben, die später noch um die Bereichsbezeichnung Rettungsmedizin deren Etablierung er selbst maßgeblich unterstützte - ergänzt wurde.

Klinisch bekleidete er in den Hamburger Krankenhäusern Ochsenzoll (Klinikum Nord) und Barmbek Direktions- und ärztliche Führungsaufgaben. 1998 gründete er das Hamburger Institut für Notfallmedizin (IfN), das er bis zuletzt leitete. Im Asklepios-Konzern war Prof. Moecke als Konzernbereichsleiter Medizin \& Wissenschaft tätig, er war Vertreter der leitenden Angestellten im Aufsichtsrat der Asklepios Kliniken Hamburg und führte bis zuletzt die von ihm gegründete Ärzteakademie in Hamburg. 2011 erhielt er die Ehrenprofessur der Hochschule für Angewandte Wissenschaften Hamburg, 2013 das Verdienstkreuz am Bande des Verdienstordens der Bundesrepublik Deutschland.

In den letzten Jahren widmete er sich in unzähligen Zeitschriftenartikeln, Büchern, Buchbeiträgen und Fachvorträgen insbesondere dem Qualitäts- und Risikomanagement in der Akutmedizin, vielen Aspekten der angewandten Patientensicherheit und der Simulation in Notfall- und Rettungsmedizin (s. auch http://www.moecke.eu).
Seine geniale Fähigkeit, Menschen zu begeistern sowie aktive und produktive Netzwerke zu knüpfen, war beeindruckend. Er prägte als hervorragender Mediziner, mutiger wie kreativer Innovator und zugleich uneitler Impulsgeber seine und unsere tägliche Arbeit. Er hat seine Visionen nicht verkündet, sondern verwirklicht.

Unser Mitgefühl gilt seiner Familie und insbesondere seiner Frau Corinna, die ihm in guten und schweren Zeiten stets zur Seite stand.

Er fehlt sehr. Wir werden ihm ein ehrendes Gedenken bewahren.

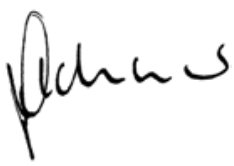

\section{K. Lackner}

Tegernseer Tal

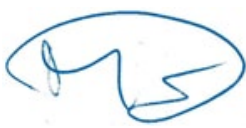

M. Reng

Poikam

\section{Korrespondenzadresse}

Prof. Dr. med. C.K. Lackner

Drees \& Sommer AG,

Geisenhausenerstr. 17, 81379, München

christian.lackner@dreso.com

\section{Ärzte erwarten Aufnahme von Apps in die Leitlinien}

43,8\% der Ärzte in Deutschland erwarten, dass therapieunterstützende Apps innerhalb der nächsten zehn Jahre in die Leitlinien aufgenommen werden. Zu diesem Ergebnis kommt die aktuelle eHealth-Studie 2015 aus der Studienreihe „Ärzte im Zukunftsmarkt Gesundheit" der Stiftung Gesundheit. Noch im Vorjahr hatten mehr als zwei Drittel der befragten Ärzte bezweifelt, dass Apps in Zukunft überhaupt den Gesundheitszustand ihrer Patienten überwachen könnten oder sich in dieser Funktion durchsetzen würden. In der Vergangenheit hatten sich Ärzte bei diesem Thema sehr kritisch und zurückhaltend bis ablehnend gezeigt. Die initialen Berührungsängste mit den neuen Medien und Möglichkeiten haben sie offenbar nun aber abgelegt. Dies betrifft nicht nur jüngere Ärzte, bei denen dies thematisch bedingt zu erwarten gewesen wäre, sondern ganz im Gegenteil: Die Altersstruktur der Ärzte, die sich an der Studie beteiligt haben, entspricht weitgehend den Anteilen in der Gesamtärzteschaft, wobei die Altersgruppen über 51 Jahren sogar überproportional vertreten waren. Die aktuelle Studie sowie alle bisherigen Ausgaben der Studienreihe „Ärzte im Zukunftsmarkt Gesundheit" sind auf der Website der Stiftung Gesundheit zugänglich:

Quelle: www.stiftung-gesundheit.de/ stiftung/studien.htm 